\title{
Central Radio Propagation Laboratory Exponential Reference Atmosphere
}

\author{
B. R. Bean and G. D. Thayer
}

(June 11, 1959)

\begin{abstract}
The background and development of an exponential model of atmospheric radio refractivity, the "C.R.P.L. Exponential Reference Atmosphere" is outlined. A set of ray tracings for the model is presented in the form of tables of refraction variables for the complete range of observed values of surface refractive index. A detailed analysis of the accuracy of the ray tracing and tabulation methods is made for these tables. The variables are presented as numbers between one and ten multiplied by the appropriate power of ten, thus maintaining a maximum number of significant figures. The tables may be used for the solution of practical refraction problems involving elevation angle errors, range errors, and similar quantities.
\end{abstract}

\section{Introduction}

Some exponential models of atmospheric radio refractivity have been introduced recently to replace the standard $4 / 3$ earth treatment for applications involving long-range transmission paths, forward scatter predictions, or radar tracking at high altitudes $\left[1,2,3,4,5,6,{ }^{2}\right.$ One of these models, developed at the Central Radio Propagation Laboratory and called the "C.R.P.L. Exponential Reference Atmosphere" has been adopted for use by the National Bureau of Standards in predictions of refraction phenomena.

In the Monograph the background of the exponential reference atmosphere is outlined and a set of radio ray tracings presented. The ray tracings may be used for the solution of many problems involving refraction.

\section{Background}

In dealing with refraction problems it is convenient to use the radio refractivity defined by

$$
N \equiv(n-1) \times 10^{6}
$$

rather than the radio refractive index, $n$. The basic predictor of refraction effects used in the exponential reference atmosphere is the surface value of refractivity at the transmitting point, $N_{s}$. The reasons for this become clear upon examination of the normal, or average, vertical $N$-structure of the atmosphere.

There is an observed high correlation between the mean value of $\Delta N$, the drop in $N$ from the surface to a height of $1 \mathrm{~km}$, and mean value of $N_{s}$. This is expressed by

$$
-\Delta N=7.32 \exp \left\{0.005577 N_{s}\right\}
$$

\footnotetext{
1 Digest of NBS Monograph (in press).

2 Figures in brackets indicate the literature references at the end of this paper.
}

which has been derived from 6 yr means for 45 U.S weather stations representing all types of climatic conditions encountered in the United States. Thus it is evident that on the average the decay of $N$ with height may be expressed as a function of the surface value of $N$, at least over the lower portion of the earth's atmosphere which is the critical portion when considering refraction effects.

It is also found that the average refractivity structure of the atmosphere follows a nearly exponential decay with height, the value of $N$ becoming insignificantly small at altitudes over $50 \mathrm{~km}$.

For these reasons the exponential reference atmosphere was defined as that family of exponential refractivity profiles where the rate of $N$ decay with height is uniquely specified by the value of $N_{s}$ in accordance with eq (1). The exponential reference atmosphere is thus represented by the following set of equations:

$$
\begin{aligned}
& N=N_{s} \exp \left\{-c_{e} h\right\} \\
& c_{e}=\ln \left\{N_{s} /\left(N_{s}+\Delta N\right)\right\}
\end{aligned}
$$

with $\Delta N$ determined from (1) above.

It is found that several important advantages accrue from this particular choice of model, among which are:

1. The $N$-profiles involved prove to be a fairly good representation of average $N$-structure over the first $3 \mathrm{~km}$ above the surface, a critical range for refraction effects at low angles to the horizon.

2 . The resulting relationship between the $N$ gradient near the surface and $N_{s}$ is found to be very close to the observed average relationship being within \pm 3 percent over the normally encountered range of $N_{s}$ (from 300 to 365 for sea level, temperate zone stations).

3. The resulting profiles and their associated gradients are continuous functions of height and are in reasonably good agreement with the actual 
observed refractivity at all heights below the ionosphere.

An illustration of the effectiveness of the exponential reference atmosphere in predicting average refraction effects is given by figure 1 , where values of angular ray bending are plotted against height for rays starting at zero elevation angle. The agreement hetween the average bending for 22 climatically diverse conditions and the average of the values predicted for each climatic condition by the exponential reference atmosphere is seen to be quite good, particularly as regards the form of the $\tau$ versus height curve. Note, however, that the bending versus height predicted by the $4 / 3$ earth model is markedly in disagreement with the observed values for most heights.

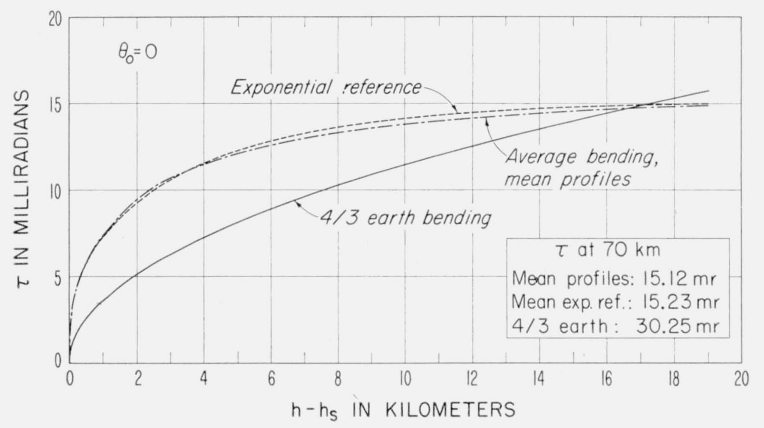

Figure 1. Comparisons of long-term mean bending with (a) the average of the values predicted by the appropriate exponential reference atmospheres and with (b) those predicted for the $4 / 3$ earth's atmosphere.

The preceding material is intended only as a background for the development of the exponential reference atmosphere; a more detailed treatment may be found in reference [5].

The remainder of the Monograph is devoted to a detailed analysis of the ray-tracing theory used to calculate refraction variables for the exponential reference atmosphere, together with an analysis of the actual method of calculation. The extensive tables of refraction variables are presented in an appendix to the Monograph.

\section{Tables of the Exponential Reference Atmosphere}

The tables of refraction variables for the "Exponential Reference Atmosphere" are presented in the form of ray tracings for profiles with $N_{s}=200.0$, $252.9,289.0,313.0,344.5,377.2,404.9$, and 450.0 . The uneven values of $N_{s}(252.9,344.5,377.2,404.9)$ were chosen to have even values of $\Delta N(30.0,50.0$, $60.0,70.0)$. The ray tracings are presented for initial elevation angles of $0,0.5,1,2,4,8,15,30,65$, $100,200,400$, and 900 milliradians, the refraction variables being tabulated at constant heights (of the ray path above the surface) consisting of 0.01 , $0.02,0.05,0.1,0.2,0.305(1,000 \mathrm{ft}), 0.5,0.7,1.0$, $1.524(3,000 \mathrm{ft}), 2.0,3.048(10,000 \mathrm{ft}), 5.0,7.0,10.0$,
$20.0,30.48(100,000 \mathrm{ft}), 50.0,70.0,90.0,110.0,225.0$, 350.0 , and $475.0 \mathrm{~km}$. Thus there are 104 ray tracings, each tabulated at 24 heights.

The following refraction variables are tabulated at each height: the local elevation angle, $\theta$; the distance, $d$, subtended on the earth's surface by the ray path from a height of zero to the tabulated height; the angular ray bending, $\tau$; the elevation angle error, $\epsilon$; the slant range, $R_{o}$, (straight line distance from origin to the point on the ray path being considered); the range error, $\Delta R$, (the difference between the geometric length of the curved ray path, $R=\int d R$, and the slant range); the radar range error, $\Delta R_{e}$, (the difference between the electrical ray path, $R_{e}=$ $\int n d R$, and the slant range); $\Delta h$, the difference between the tabulated height and the height of a ray calculated with the $4 / 3$ earth assumption at the same distance along the earth's surface; and $\Delta \theta$, the difference between $\theta$ for the exponential reference atmosphere and $\theta$ for the $4 / 3$ earth ray at the same height. All entries are given in a form of floating point notation where all numbers are between 1 and 10 multiplied by the appropriate power of 10 , and the number $\pm N . N \times 10^{ \pm m}$ is printed as $\pm N . N \pm m$, where the second sign is the sign of the exponent of ten.

Four refraction quantities which are not listed can be easily obtained from the tables: the angular distance subtended by the ray path at the center of the earth, $\phi=\left(d / r_{\mathrm{o}}\right)$ where $r_{\mathrm{o}}$ is the assumed radius of the earth at the ray path origin; the difference between the elevation angle of the ray path and the elevation angle of the slant range "path", $\delta=\tau-\epsilon$; the geometric ray path length, $R=R_{0}+\Delta R$; the electrical ray path length, $R_{e}=R_{0}+\Delta R_{e}$.

The elevation angles given in the tables are subject to a maximum error of $\pm 0.00005 \mathrm{mr}$; the values of $\tau$ and $\epsilon$ are subject to a maximum error of \pm 0.001 $\mathrm{mr}$; the values of the different ranges and range errors are subject to a maximum error of $\pm 5 \mathrm{~m}$, in particular the range errors are assumed to have a maximum error of \pm 0.5 percent or $\pm 0.1 \mathrm{~m}$, whichever is larger.

An example of the refraction tables contained in the appendix to the Monograph is given in the following table for the $N_{s}=313$ profile, $\theta_{0}=0$ (313 is the average value of $N_{s}$ determined from nearly two million weather observations taken at 45 different weather stations throughout the United States).

As an example of the calculation of the four unlisted quantities previously mentioned, one obtains for the height of $1 \mathrm{~km}$ :

$$
\begin{aligned}
& \phi=\frac{d}{r_{0}}=\frac{133.073}{6373.2284}=0.0208800 \mathrm{radian} \\
& \delta=\tau-\epsilon=5.71671-2.9263=2.7904 \mathrm{mr} \\
& R=R_{\mathrm{o}}+\Delta R=133.08495+0.00018=133.08513 \mathrm{~km} \\
& R_{e}=R_{\mathrm{o}}+\Delta R_{e}=133.08495+0.03993=133.12488 \mathrm{~km}
\end{aligned}
$$


TABLE 1. Example of tabulated refraction variables for $\theta_{0}=0$ and $N_{s}=313$

\begin{tabular}{|c|c|c|c|c|c|c|c|c|c|c|c|}
\hline $\begin{array}{c}\text { Sur- } \\
\text { face } N^{-}\end{array}$ & $\begin{array}{l}\text { Init } \\
\text { theta }\end{array}$ & Height & Delta $H$ & Theta & $\begin{array}{l}\text { Delta } \\
\text { theta }\end{array}$ & Distance & Tau & $\begin{array}{l}\text { Error } \\
\text { angle }\end{array}$ & Slant range & Delta $R$ & $\begin{array}{c}\text { Delta } \\
R-E\end{array}$ \\
\hline 313.0 & 0.0 & 0.010 & $-5.1424-4$ & 1. 49613 & 7. $6990-2$ & 1. 336781 & 6. 01363-1 & 3. $0068-1$ & 1. 33678191 & 0.000 & 4. 181- 3 \\
\hline 313.0 & 0 & .020 & $-1.0273-3$ & 2.11617 & 1. 0849-1 & 1.890441 & 8. $50046-1$ & 4. $2516-1$ & 1. 89044491 & 1. $000-6$ & 5. $912-3$ \\
\hline 313.0 & 0 & .050 & $-2.5545-3$ & 3. 34743 & 1. $6960-1$ & 2.988651 & 1. 34194 & 6. $7171-1$ & 2. $9886667 \quad 1$ & 3. $000-6$ & $9.334-3$ \\
\hline 313.0 & 0 & .100 & $-5.0557-3$ & 4. 73735 & $3521-1$ & 4. 225501 & 1. 89273 & 9. $4849-1$ & 4. 22554651 & 6. $000-6$ & 1. $317-2$ \\
\hline 313.0 & 0 & .200 & $-9.9458-3$ & 6.70910 & 3. $2035-1$ & 5. 973381 & 2. 66351 & 1. 3382 & 5. 97349001 & $2.000-5$ & 1. $854-2$ \\
\hline 313.0 & .0 & .305 & $-1.4835-2$ & 8. 29831 & 3. $7788-1$ & 7. 372721 & 3. 26995 & 1. 6474 & 7. 37291901 & $3.500-5$ & 2. $278-2$ \\
\hline 313.0 & 0 & .500 & $-2.3345-2$ & 1.06519 1 & 4. $4631-1$ & 9.430921 & 4. 14574 & 2.0975 & 9.43134201 & 6. $600-5$ & $2.890-2$ \\
\hline 313.0 & .0 & .760 & $-3.1325-2$ & 1. 263731 & 4. $8205-1$ & 1.114832 & 4. 85517 & 2. 4678 & 1. 11490532 & 1. $000-4$ & 3. $387-2$ \\
\hline 313.0 & .0 & 1. 000 & $-4.2035-2$ & 1.516331 & $4.9670-1$ & 1.330732 & 5. 71671 & 2. 9263 & 1. 33084952 & 1. $800-4$ & 3. $993-2$ \\
\hline 313.0 & .0 & 1. 524 & $-5.6544-2$ & 1.884051 & $4.4550-1$ & 1. 638852 & 6.87418 & 3.5600 & 1. 63907852 & $3.100-4$ & 4. $812-2$ \\
\hline 313.0 & .0 & 2. 000 & $-6.5784-2$ & 2. 170291 & 3. 4521-1 & 1. 873562 & 7. 69459 & 4. 0267 & 1. 87390272 & 4. $600-4$ & 5. $398-2$ \\
\hline 313.0 & .0 & 3. 048 & $-7.3319-2$ & 2. 709271 & 7. $6253-3$ & 2. 302892 & 9.04109 & 4. 8367 & 2. 30352142 & $7.800-4$ & 6. $369-2$ \\
\hline 313.0 & .0 & 5.000 & $-4.6267-2$ & 3.531551 & $-8.6362-1$ & 2.927852 & 1. 062431 & 5. 9039 & $2.9291690 \quad 2$ & 1. $400-3$ & 7. $539-2$ \\
\hline 313.0 & 0 & 7.000 & 2. $4347-2$ & 4. 239941 & -1.8936 & 3. 442032 & 1. 160821 & 6. 6859 & 3. 44421782 & 2. $000-3$ & 8. $284-2$ \\
\hline 313.0 & .0 & 10.000 & 1. $9795-1$ & 5. 154711 & -3.5381 & 4. 079632 & 1. 246481 & 7. 5264 & 4. 08335982 & 2. $790-3$ & 8. $960-2$ \\
\hline 313.0 & .0 & 20.000 & 1. 1676 & 7.530201 & -8.7861 & 5. 652272 & 1. 338571 & 9. 0531 & 5. 66281652 & 4. $460-3$ & 9. $801-2$ \\
\hline 313.0 & .0 & 30.480 & 25569 & $9.43972 \quad 1$ & -1.34391 & 6.879532 & 1. 354701 & 9. 8441 & 6.89935952 & 5.47 & 1. $003-1$ \\
\hline 313.0 & .0 & 50.000 & 5.6578 & 1. 223372 & $-2.0398 \quad 1$ & 8. 662382 & 1. 358071 & 1. 06151 & 8. 70398202 & $6.520-3$ & 1. $017-1$ \\
\hline 313.0 & .0 & 70.000 & 9.2212 & 1.454172 & $-2.6167 \quad 1$ & 1.013343 & 1. $35824 \quad 1$ & $1.1051 \quad 1$ & 1.02022523 & 7. $100-3$ & 1. $024-1$ \\
\hline 313.0 & 0 & 90.000 & 1. 30251 & 1. 652102 & -3.11151 & 1. 139493 & 1. 358251 & 1. 13351 & 1. 14950903 & $7.500-3$ & 1. $028-1$ \\
\hline 313.0 & .0 & 110.000 & 1. 70031 & 1. 827872 & -3.55091 & 1. 251513 & 1. 358251 & 1. 15401 & 1. 26502933 & 7. $800-3$ & 1. $031-1$ \\
\hline 313.0 & .0 & 250.000 & 4. $7697 \quad 1$ & 2.745202 & -5.84431 & 1.836143 & 1. 358251 & 1. 22101 & 1.88202563 & $8.600-3$ & 1. $038-1$ \\
\hline 313.0 & .0 & 350.000 & 7.16131 & 3. 231542 & -7.06011 & 2.146103 & 1. 358251 & 1. 24191 & 2. 22158783 & $8.900-3$ & 1. $042-1$ \\
\hline 313.0 & .0 & 475.000 & 1.03212 & 3. 738442 & -8.32741 & 2. 469153 & 1. 358251 & 1. 25841 & 2.58751533 & $9.000-3$ & 1. $042-1$ \\
\hline
\end{tabular}

\section{References}

[1] M. Schulkin, Average radio-ray refraction in the lower atmosphere, Proc. I.R.E. 40, 554 (1952).

[2] L. J. Anderson, Tropospheric bending of radio waves, Trans. American Geophys. Union, 39, 208 (1958).

[3] J. R. Bauer, W. C. Mason, F. A. Wilson, Radio refraction in a cool exponential atmosphere. Lincoln Labs. Tech. Rept. No. 186 (Aug. 27, 1958).

[4] G. Millington, Propagation at great heights in the atmosphere, Marconi Rev. 21, 143 (Fourth Quart, 1958).
[5] B. R. Bean and G. D. Thayer, On models of the atmospheric radio refractive index, Proc. I.R.E. 47, 740 (1959).

[6] B. R. Bean, Atmospheric bending of radio waves, paper presented at Intern. Conf. on Electromagnetic Wave Propagation, Liege, Belgium, Oct. 1958; to be published by the Academic Press.

Boulder, Colo.

(Paper 63D3-28) 